\title{
Estimates on the Lower Bound of the Eigenvalue of the Smallest Modulus Associated with a General Weighted Sturm-Liouville Problem
}

\author{
Mervis Kikonko ${ }^{1,2}$ and Angelo Bernado Mingarelli ${ }^{3}$ \\ ${ }^{1}$ Department of Engineering Sciences and Mathematics, Luleå University of Technology, 97187 Luleå, Sweden \\ ${ }^{2}$ Department of Mathematics and Statistics, University of Zambia, P.O. Box 32379, Lusaka, Zambia \\ ${ }^{3}$ School of Mathematics and Statistics, Carleton University, Ottawa, ON, Canada K1S 5B6
}

Correspondence should be addressed to Mervis Kikonko; mervis.kikonko@ltu.se

Received 6 June 2016; Accepted 11 August 2016

Academic Editor: Yoshikazu Giga

Copyright (C) 2016 M. Kikonko and A. B. Mingarelli. This is an open access article distributed under the Creative Commons Attribution License, which permits unrestricted use, distribution, and reproduction in any medium, provided the original work is properly cited.

We obtain a lower bound on the eigenvalue of smallest modulus associated with a Dirichlet problem in the general case of a regular Sturm-Liouville problem. The main motivation for this study is the result obtained by Mingarelli (1988).

\section{Introduction}

In this paper, we derive some lower bound for an eigenvalue of the smallest modulus (not necessarily unique) corresponding to the problem

$$
\begin{aligned}
-u^{\prime \prime}(x)+q(x) u(x) & =\lambda r(x) u(x), \quad a \leq x \leq b, \\
u(a) & =u(b)=0,
\end{aligned}
$$

consolidating the results obtained by Mingarelli [1]. In the paper a lower bound for the eigenvalue of the smallest modulus was obtained under the assumptions that $q, r$ : $[a, b] \rightarrow \mathbb{R}, r \in L^{\infty}(a, b), q \in L^{\infty}(a, b),|r(x)|>0$ a.e. on $(a, b)$, and that $q(x)$ and $r(x)$ take on both positive and negative values on $(a, b)$. The parameter $\lambda \in \mathbb{C}$ is called an eigenvalue and corresponding function $u$ not identically zero on $[a, b]$ is called an eigenfunction. The eigenfunctions corresponding to real eigenvalues can be labeled in such a way that $u_{n}(x)=u\left(x, \lambda_{n}\right)$ and $u_{n}(x)$ has $|n|$ zeros in $(a, b)$, $n=\ldots,-2,-1,0,1,2, \ldots$. Therefore the eigenvalue of the smallest modulus will be labeled $\lambda_{0}$ and the corresponding eigenfunction $u_{0}(x)$ has no zero in $(a, b)$. In this paper we assume in general that $\lambda=0$ is not an eigenvalue. Our studies here are an extension of the results in [1] to the case where the assumption on the coefficient function $q(x)$ is replaced by the more general assumption that $q \in L^{1}(a, b)$. We use the Fredholm integral operator associated with (1)-(2) in the Hilbert space $L_{|r|}^{2}(a, b) \equiv \mathscr{H}$ and use the estimates on its norm and the $\mathscr{H}$-norms of solutions of a Cauchy problem related to (1)-(2). We consider the general weighted Sturm-Liouville problem which is the case in which the coefficient functions $q(x)$ and $r(x)$ have no sign restrictions imposed on them. The weight function $r(x)$ plays a critical role in the nature of $\lambda_{0}$. In the next subsection we give a brief outline of the three different cases that arise as one varies the signs of the weight function $r(x)$ and the coefficient function $q(x)$.

(1) Preliminary Results. When $r(x)>0$ and $q(x)$ takes on both positive and negative values on the interval $(a, b)$, problem (1)-(2) is right definite and only one sequence of real eigenvalues $\lambda_{n}$ exists with $\lambda_{n} \rightarrow \infty$ as $n \rightarrow \infty$. For more information on this case, we refer the interested reader to [2-4] and so forth and the references within. Particularly, Everitt et al. [2, theorem 0] outline oscillation properties of eigenfunctions of the right definite problem. In the right definite case $\lambda_{0}$ is the smallest eigenvalue with the corresponding eigenfunction having no zero in the interval $(a, b)$.

When $q(x) \geq 0$ a.e. and $r(x)$ takes on both signs on sets of positive Lebesgue measure, the problem is left definite. 
For more details see, for example, [4-6] and the references therein. In this case two sequences of real eigenvalues $\lambda_{n}^{ \pm}$ exist where $\lambda_{n}^{ \pm} \rightarrow \pm \infty$, one positive and the other negative, and $\lambda_{0}$ is then the first positive (and or negative) eigenvalue (whose eigenfunction is positive on $(a, b)$ ) labeled according to a Sturm oscillation theorem.

When $q(x)<0$ a.e and $r(x)$ changes sign, problem (1)-(2) is nondefinite (or indefinite). In this case, nonreal eigenvalues may exist and so $\lambda_{0}$ may be either real or nonreal. In the nondefinite case, if $\lambda_{0}$ is real, then the corresponding eigenfunction can have any number of zeros on the interval $(a, b)$ in contrast with the other two cases. There is a lot of literature covering this case (see, e.g., $[4,7,8]$ and references therein). More studies are carried out on existence and estimation of nonreal eigenvalues in the recent papers [913 ] and so forth. In some of these papers upper and lower bounds on the nonreal eigenvalues of indefinite SturmLiouville problems are estimated and the existence of nonreal eigenvalues is discussed in others.

The discreteness of the spectrum for problem (1)-(2) and the assumption that $\lambda=0$ is not an eigenvalue of the problem guarantees the existence of such an eigenvalue $\lambda_{0}$. Moreover, this eigenvalue is not unique, since there exist problems where the real spectrum is symmetric about zero in the left definite case. In fact even when nonreal eigenvalues exist, they appear in conjugate pairs; hence the nonreal spectrum is symmetric about the $x$-axis.

The assumption that $\lambda=0$ is not an eigenvalue of problem (1)-(2) is equivalent to the fact that the problem consisting of the equation

$$
-y^{\prime \prime}(x)+q(x) y(x)=0
$$

and the boundary conditions (2) admits a unique Green's function, $G(x, t)$, defined and continuous over $I \equiv[a, b] \times$ $[a, b]$. We define the inner product on $\mathscr{H}$ by

$$
(f, g)=\int_{a}^{b} f(x) \overline{g(x)}|r(x)| d x, \quad f, g \in \mathscr{H},
$$

and the associated norm $\|\cdot\|$ is given by

$$
\|f\|=\left(\int_{a}^{b}|f(x)|^{2}|r(x)| d x\right)^{1 / 2} .
$$

Green's function for problem (3)-(2) takes the form

$$
G(x, t)= \begin{cases}\frac{y(x) z(t)}{y(b)}, & \text { if } x \in[a, t], \\ \frac{y(t) z(x)}{y(b)}, & \text { if } x \in[t, b],\end{cases}
$$

where $y, z$ are (real) linearly independent solutions of (3) satisfying the initial conditions

$$
\begin{gathered}
y(a)=0 ; \\
y^{\prime}(a)=1, \\
z(b)=0 ; \\
z^{\prime}(b)=1,
\end{gathered}
$$

respectively. In a lot of literature (see. e.g.. [14]) it is shown that the spectral problem (1)-(2) can be reduced to a single eigenvalue equation

$$
T u=\mu r(x) u,
$$

where the operator $T$ is defined by

$$
(T f)(x)=\int_{a}^{b} G(x, t) f(t) r(t) d t
$$

on $\mathscr{H}$. Here $G$ is Green's function associated with problem (1)(2), $|r(x)|>0$, and $\mu=-1 / \lambda$. That is, $u$ is an eigenfunction of problem (1)-(2) if and only if $u$ is an eigenfunction of $T$ associated with the eigenvalue $-1 / \lambda$.

\section{The Main Results}

We start by stating two important lemmas from [1] whose results we will use in proving the results in this section. We present the lemmas without proof and refer the interested reader to the cited paper.

Lemma 1. The linear operator $T$ defined by (10) maps $\mathscr{H}$ into $\mathscr{H}$ and is a bounded (compact but not necessarily self-adjoint) operator on $\mathscr{H}$ whose operator norm, $\|T\|$, is given by

$$
\|T\|=\left(\iint_{I}|G(x, t)|^{2}|r(x)||r(t)| d x d t\right)^{1 / 2} .
$$

In the same paper it is also shown that an eigenvalue $\lambda_{0}$ of problem (1)-(2) having the smallest modulus admits the lower bound

$$
\left|\lambda_{0}\right| \geq \frac{1}{\|T\|} .
$$

Lemma 2. An eigenvalue $\lambda_{0}$ of (1)-(2) of the smallest modulus satisfies

$$
\left|\lambda_{0}\right| \geq|y(b)|(\sqrt{2}\|y\|\|z\|)^{-1},
$$

where $y, z$ are given in (6), (7), and (8), and $\|y\|,\|z\|$ are their respective $\mathscr{H}$-norms.

We now give a variant of lemma 4 in [1].

Lemma 3. Let $y(x, q)$ denote the solution of the Cauchy problem

$$
\begin{aligned}
& -y^{\prime \prime}(x)+q(x) y(x)=0, \quad a \leq x \leq b, \\
& y(a)=0 ; \\
& y^{\prime}(a)=1, \\
& B(c)=\left\{q:[a, b] \longrightarrow \mathbb{R}, q \in L^{1}(a, b),\|q\|_{1}=c\right\} .
\end{aligned}
$$

Then for fixed $x$ in $[a, b]$,

$$
\sup _{q \in B(c)}|y(x, q)| \leq(x-a) e^{c(x-a)} .
$$


Similarly, if $z(x, q)$ denotes the solution of (14) satisfying

$$
\begin{gathered}
z(b)=0 ; \\
z^{\prime}(b)=1,
\end{gathered}
$$

then for fixed $x$ in $[a, b]$,

$$
\sup _{q \in B(c)}|z(x, q)| \leq(b-x) e^{c(b-x)} .
$$

Proof. To prove (17), we use the integral representation of (14)-(15) which is given by

$$
y(x)=x-a+\int_{a}^{x}(x-s) q(s) y(s) d s .
$$

From this we get the Neumann series expansion of $y$ which is given by the terms below.

$$
\begin{aligned}
& A_{0}=x-a, \\
& A_{1}=\int_{a}^{x}\left(x-s_{1}\right)\left(s_{1}-a\right) q\left(s_{1}\right) d s_{1}, \\
& \left|A_{1}\right| \leq \frac{(x-a)^{2}}{2^{2}}\|q\|_{1}, \\
& A_{2}=\int_{a}^{x} \int_{a}^{s_{1}}\left(x-s_{1}\right)\left(s_{1}-s_{2}\right)\left(s_{2}-a\right) q\left(s_{1}\right) \\
& \cdot q\left(s_{2}\right) d s_{2} d s_{1}, \\
& \left|A_{2}\right| \leq \frac{(x-a)^{3}}{3^{3}}\|q\|_{1}^{2}, \\
& A_{3}=\int_{a}^{x} \int_{a}^{s_{1}} \int_{a}^{s_{2}}\left(x-s_{1}\right)\left(s_{1}-s_{2}\right)\left(s_{2}-s_{3}\right)\left(s_{3}-a\right) \\
& \cdot q\left(s_{1}\right) q\left(s_{2}\right) q\left(s_{3}\right) d s_{3} d s_{2} d s_{1}, \\
& \left|A_{3}\right| \leq \frac{(x-a)^{4}}{4^{4}}\|q\|_{1}^{3} .
\end{aligned}
$$

Generally,

$$
A_{n}=\int_{R_{n}} \prod_{i=0}^{n}\left(s_{i}-s_{i+1}\right) Q(s) d s, \quad n \geq 1,
$$

where

$$
\begin{aligned}
& R_{n}=\left\{\left(s_{1}, s_{2}, \ldots, s_{n}\right): a \leq s_{1} \leq x, a \leq s_{i} \leq s_{i-1}, i\right. \\
& \quad=2,3,4, \ldots, n\}, \\
& Q(s)=Q\left(s_{1}, s_{2}, \ldots, s_{n}\right)=q\left(s_{1}\right) q\left(s_{2}\right) \cdots q\left(s_{n}\right), \\
& d s=d s_{n} d s_{n-1} \cdots d s_{1}, \\
& s_{0}=x \\
& s_{n+1}=a .
\end{aligned}
$$
bound

If $\|q\|_{1}=c$ similar working as above yields the upper

$$
\left|A_{n}\right| \leq c^{n}\left(\frac{x-a}{n+1}\right)^{n+1}, \quad n \geq 1 .
$$

Therefore we have that

$$
\begin{aligned}
|y(x)| & =\left|x-a+\sum_{n=1}^{\infty} A_{n}\right| \leq|x-a|+\sum_{n=1}^{\infty}\left|A_{n}\right| \\
& \leq x-a+\sum_{n=1}^{\infty} c^{n}\left(\frac{x-a}{n+1}\right)^{n+1} \\
& =(x-a) \sum_{n=0}^{\infty} \frac{c^{n}(x-a)^{n}}{(n+1)^{n+1}} \\
& <(x-a) \sum_{n=0}^{\infty} c^{n} \frac{(x-a)^{n}}{n !}
\end{aligned}
$$

and the series

$$
\sum_{n=0}^{\infty} c^{n} \frac{(x-a)^{n}}{n !}
$$

converges, so the Neumann series converges too. This yields that

$$
|y(x)|<(x-a) e^{c(x-a)},
$$

and the bound in (17) is established. by

Similarly, the integral representation of (14)-(18) is given

$$
z(x)=x-b+\int_{x}^{b}(s-x) q(s) y(s) d s .
$$

The corresponding Neumann series is given by the terms

$$
\begin{aligned}
& B_{0}=b-x, \\
& B_{n}=\int_{R_{n}} \prod_{i=0}^{n}\left(s_{i}-s_{i+1}\right) Q(s) d s, \quad n \geq 1,
\end{aligned}
$$

where

$$
\begin{aligned}
& R_{n}=\left\{\left(s_{1}, s_{2}, \ldots, s_{n}\right): x \leq s_{1} \leq b, a \leq s_{i} \leq s_{i-1}, i\right. \\
& \quad=2,3,4, \ldots, n\}, \\
& Q(s)=Q\left(s_{1}, s_{2}, \ldots, s_{n}\right)=q\left(s_{1}\right) q\left(s_{2}\right) \cdots q\left(s_{n}\right), \\
& d s=d s_{n} d s_{n-1} \cdots d s_{1}, \\
& s_{0}=b, \\
& s_{n+1}=x, \\
& \left|B_{n}\right| \leq c^{n}\left(\frac{b-x}{n+1}\right)^{n+1}, \quad n \geq 1 .
\end{aligned}
$$

Hence,

$$
\begin{aligned}
|z(x)| & =\left|b-x+\sum_{n=1}^{\infty} B_{n}\right| \leq|b-x|+\sum_{n=1}^{\infty}\left|B_{n}\right| \\
& \leq b-x+\sum_{n=1}^{\infty} c^{n}\left(\frac{b-x}{n+1}\right)^{n+1} \\
& <(b-x) \sum_{n=0}^{\infty} c^{n} \frac{(b-x)^{n}}{n !}
\end{aligned}
$$


which yields that

$$
|z(x)|<(b-x) e^{c(b-x)},
$$

and the bound in (19) is established.

The results in Lemma 3 lead to the estimation of the $\mathscr{H}$ norm of the solutions $y, z$, which leads to the following result.

Lemma 4. Let $y, z$ denote the two linearly independent solutions of (14) satisfying (15) and (18), respectively. Then

$$
\begin{aligned}
& \sup _{q \in B(c)}\|y(x, q)\| \\
& \leq\left(\int_{a}^{b}\left((x-a) e^{c(x-a)}\right)^{2}|r(x)| d x\right)^{1 / 2}, \\
& \sup _{q \in B(c)}\|z(x, q)\| \\
& \leq\left(\int_{a}^{b}\left((b-x) e^{c(b-x)}\right)^{2}|r(x)| d x\right)^{1 / 2} .
\end{aligned}
$$

Proof. We prove the results by calculating the $\mathscr{H}$-norms of $y$ and $z$, and use the bounds in Lemma 3 as shown below.

$$
\begin{aligned}
\|y(x, q)\| & =\left(\int_{a}^{b}|y(x, q)|^{2}|r(x)| d x\right)^{1 / 2} \\
& <\left(\int_{a}^{b}(x-a)^{2} e^{2 c(x-a)}|r(x)| d x\right)^{1 / 2} .
\end{aligned}
$$

Taking the supremum on $\|y(x, q)\|$ in the inequality yields the bound in (33). A similar procedure yields (34).

Applying the results in Lemma 4 to the lower bound in (13), we get the main result which is a variant of theorem 1 in [1].

Theorem 5. Let $\|q\|_{1}=c$. Then for problem (1)-(2), an eigenvalue $\lambda_{0}$ of the smallest modulus may be estimated by

$$
\begin{array}{r}
\left|\lambda_{0}\right| \\
\geq|y(b)|\left(\sqrt{2}\left\|(x-a) e^{c(x-a)}\right\|\left\|(b-x) e^{c(b-x)}\right\|\right)^{-1}, \\
x \in(a, b),
\end{array}
$$

where $y(b)$ is the solution of the Cauchy problem (14)-(15) evaluated at $x=b$.

2.1. Examples. Here we give examples to verify if the inequality in (36) really holds. Without loss of generality, we consider the case where $q(x)=q_{0} \in \mathbb{R}$ on the interval $[-1,1]$. In this case, (36) becomes

$$
\begin{aligned}
& \left|\lambda_{0}\right| \\
& \quad \geq|y(1)|\left(\sqrt{2}\left\|(x+1) e^{c(x+1)}\right\|\left\|(1-x) e^{c(1-x)}\right\|\right)^{-1},
\end{aligned}
$$

where $c=\left(\int_{-1}^{1}\left|q_{0}\right|\right)^{1 / 2}$. The eigenvalues are found using the Maple $^{(}$package RootFinding(Analytic).
Example 1 (the case $q_{0}=-6 \pi^{2}$ and $r(x)$ changes sign). We consider the problems

$$
\begin{aligned}
y^{\prime \prime}+6 \pi^{2} y & =0, \\
y(-1) & =0, \\
y^{\prime}(-1) & =1, \\
y^{\prime \prime}+6 \pi^{2} y & =-\lambda r(x) y, \\
y(-1) & =0=y(1),
\end{aligned}
$$

where

$$
r(x)= \begin{cases}-1, & \text { if } x \in(-1,0), \\ 1, & \text { if } x \in(0,1) .\end{cases}
$$

The solution to the problem in (38) is $y(x)=\sin \sqrt{6} \pi(x+$ $1) /(\sqrt{6} \pi)$ and so

$$
y(1)=\frac{(\sin (2 \sqrt{6} \pi))}{(\sqrt{6} \pi)} .
$$

Substituting in (37) yields that $\lambda_{0} \geq 3.048 \times 10^{-11}$, and solving problem (39) gives the spectrum to be

$$
\begin{aligned}
& \ldots,-157,-67.2,-52.2,-14.3 \pm 11.81,14.3 \\
& \quad \pm 11.81,52.2,67.2,157.8, \ldots
\end{aligned}
$$

From the spectrum we see that $\lambda_{0}$ is not unique in this particular case since $|-14.3 \pm 11.8 \imath|$ and $|14.3 \pm 11.8 \imath|$ all give the smallest modulus which is 18.5. Therefore, $\left|\lambda_{0}\right|=18.5$ and (37) is satisfied.

Example 2 (the case $q_{0}=6 \pi^{2}$ and $r(x)$ changes sign). We consider the problems

$$
\begin{aligned}
-y^{\prime \prime}+6 \pi^{2} y & =0, \\
y(-1) & =0, \\
y^{\prime}(-1) & =1, \\
-y^{\prime \prime}+6 \pi^{2} y & =\lambda r(x) y, \\
y(-1) & =0=y(1),
\end{aligned}
$$

where $r(x)$ is as given in Example 1 .

The solution to the problem in (43) is $y(x)=$ $\sinh \sqrt{6} \pi(x+1) /(\sqrt{6} \pi)$ and so $y(1)=\sinh 2 \sqrt{6} \pi /(\sqrt{6} \pi)$. Substituting in (37) yields that $\lambda_{0} \geq 0.000236$, and solving problem (44) gives the spectrum to be

$$
\ldots,-137.7,-93.3,-67.6,-59.2,67.6,93.3,137.7, \ldots .
$$

From the spectrum we see that $\left|\lambda_{0}\right|=59.2$ and (37) is satisfied. 
Example 3 (the case $q_{0}=6 \pi^{2}$ and $r(x) \equiv 1$ ). Here we consider problem (43) and the problem

$$
\begin{aligned}
y^{\prime \prime}+\left(\lambda-6 \pi^{2}\right) y & =0, \\
y(-1) & =0=y(1) .
\end{aligned}
$$

Solving problem (46) gives the spectrum to be

$$
61.7,81.4,120.9,180.1, \ldots,
$$

and clearly, (37) is satisfied since $\lambda_{0}=61.7$.

\section{Conclusion}

In this paper, we undertook a study that consolidates results obtained in [1] where a lower bound for an eigenvalue $\lambda_{0}$ of smallest modulus is obtained under the assumption that $q(x) \in L^{\infty}(a, b)$. In this paper, we have considered the general case where $q(x) \in L^{1}(a, b)$ in which we obtain different bounds for the eigenvalue $\lambda_{0}$. As can be seen from Examples 1,2 , and 3 the result holds.

\section{Competing Interests}

The authors declare that there are no competing interests regarding the publication of this article.

\section{Acknowledgments}

The authors wish to thank Professor L-E Persson (Luleå University of Technology) for reading through the manuscript. They also wish to thank the Division of Mathematical Sciences and Statistics, Luleå University of Technology, Sweden, for the financial support during the research visit of the first author. Furthermore, they wish to thank the International Science Program based at Uppsala University, Sweden, for funding the Ph.D. studies and financial assistance towards the research visits by the first author. This research is supported by International Science Programme in Mathematical Sciences, Uppsala University, Sweden, and the Department of Engineering Sciences and Mathematics, Luleå University of Technology.

\section{References}

[1] A. B. Mingarelli, "Non-real eigenvalue estimates for boundary problems associated with weighted Sturm-Liouville equations," in Proceedings of the International Conference on Theory and Applications of Differential Equations, pp. 222-228, 1988.

[2] W. N. Everitt, M. K. Kwong, and A. Zettl, "Oscillation of eigenfunctions of weighted regular Sturm-Liouville problems," Journal of the London Mathematical Society, vol. 27, no. 1, pp. 106-120, 1983.

[3] F. V. Atkinson and A. B. Mingarelli, "Asymptotics of the number of zeros and of the eigenvalues of general weighted SturmLiouville problems," Journal für die Reine und Angewandte Mathematik, vol. 375-376, pp. 380-393, 1987.

[4] R. G. D. Richardson, "Contributions to the study of oscillation properties of the solutions of linear differential equations of the second order," American Journal of Mathematics, vol. 40, no. 3, pp. 283-316, 1918.

[5] A. B. Mingarelli, "A survey of the regular weighted SturmLiouville problem-the non-definite case," in Proceedings of the International Workshop on Applied Differential Equations, pp. 109-113, Beijing, China, 1986.

[6] Q. Kong, H. Wu, and A. Zettl, "Left-definite Sturm-Liouville problems," Journal of Differential Equations, vol. 177, no. 1, pp. $1-26,2001$.

[7] Q. Kong, H. Wu, A. Zettl, and M. Möller, "Indefinite SturmLiouville problems," Proceedings of the Royal Society of Edinburgh A: Mathematics, vol. 133, no. 3, pp. 639-652, 2003.

[8] A. B. Mingarelli, "Indefinite Sturm-Liouville problems," in Ordinary and Partial Differential Equations, W. N. Everitt and B. D. Sleeman, Eds., vol. 964 of Lecture Notes in Mathematics, pp. 519528, Springer, Berlin, Germany, 1982.

[9] J. Behrndt, S. Chen, F. Philipp, and J. Qi, "Bounds on non-real eigenvalues of indefinite Sturm-Liouville problems," Proceedings in Applied Mathematics and Mechanics, vol. 13, no. 1, pp. 525-526, 2013.

[10] J. Qi and S. Chen, "A priori bounds and existence of non-real eigenvalues of indefinite Sturm-Liouville problems," Journal of Spectral Theory, vol. 4, no. 1, pp. 53-63, 2014.

[11] J. Behrndt, F. Philipp, and C. Trunk, "Bounds on the nonreal spectrum of differential operators with indefinite weights," Mathematische Annalen, vol. 357, no. 1, pp. 185-213, 2013.

[12] J. Behrndt, S. Chen, F. Philipp, and J. Qi, "Estimates on the non-real eigenvalues of regular indefinite Sturm-Liouville problems," Proceedings of the Royal Society of EdinburghSection A.: Mathematics, vol. 144, no. 6, pp. 1113-1126, 2014.

[13] J. Qi, B. Xie, and S. Chen, "The upper and lower bounds on non-real eigenvalues of indefinite Sturm-Liouville problems," Proceedings of the American Mathematical Society, vol. 144, no. 2, pp. 547-559, 2016.

[14] M. A. Al-Gwaiz, Sturm-Liouville Theory and Its Applications, Springer Undergraduate Mathematics Series, Springer, London, UK, 2008. 


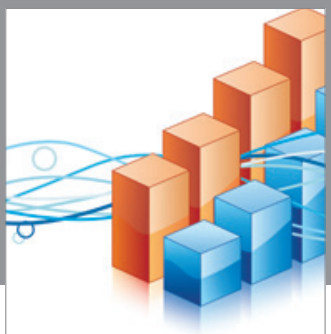

Advances in

Operations Research

vatem alat4

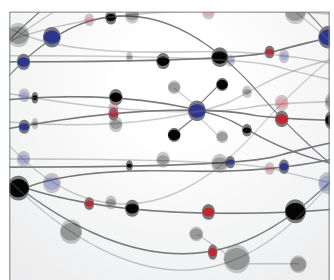

\section{The Scientific} World Journal
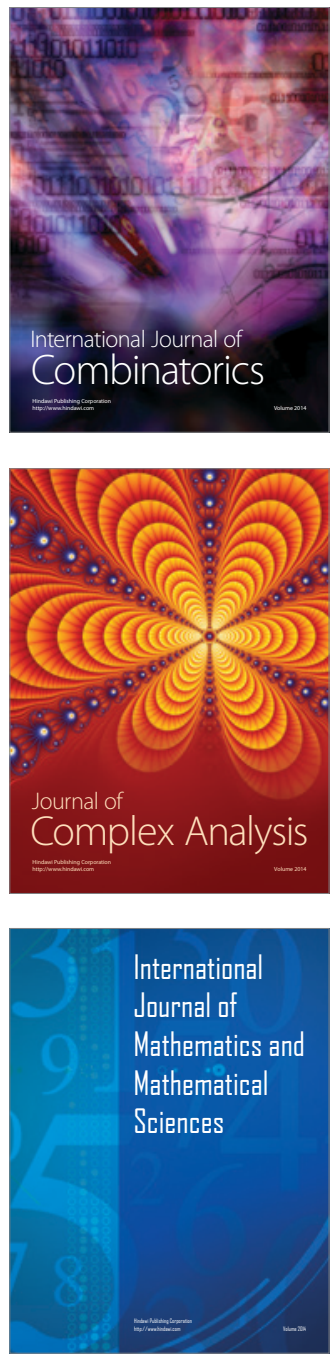
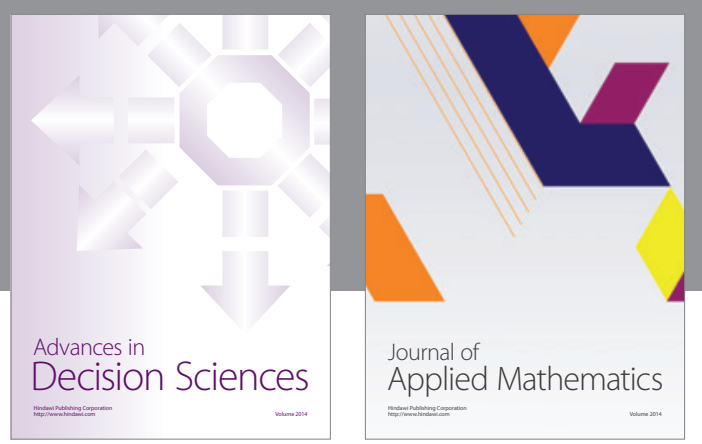

Algebra

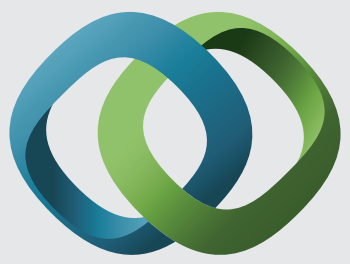

\section{Hindawi}

Submit your manuscripts at

http://www.hindawi.com
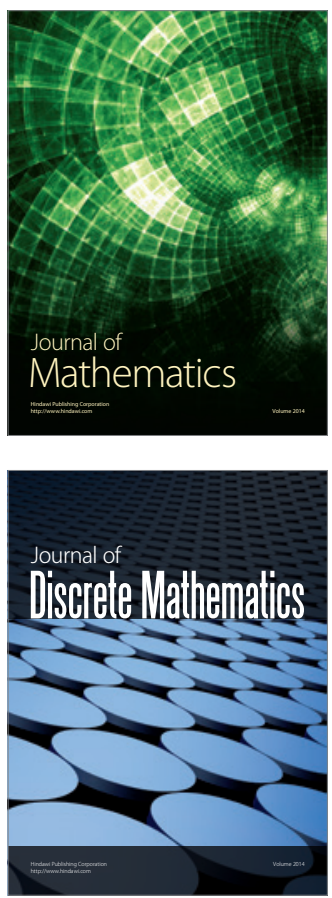

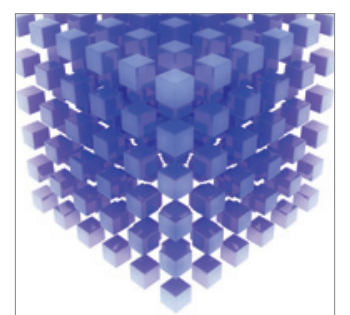

Mathematical Problems in Engineering
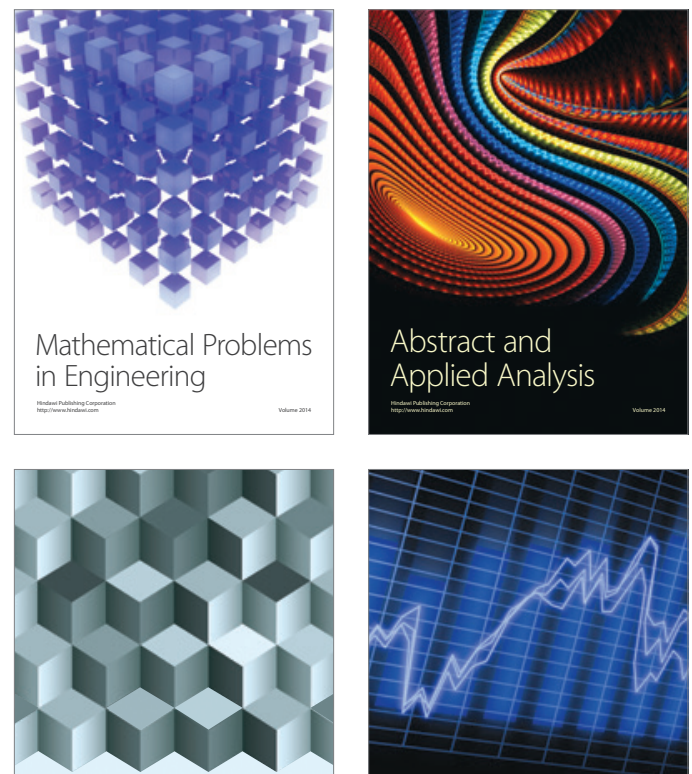

Journal of

Function Spaces

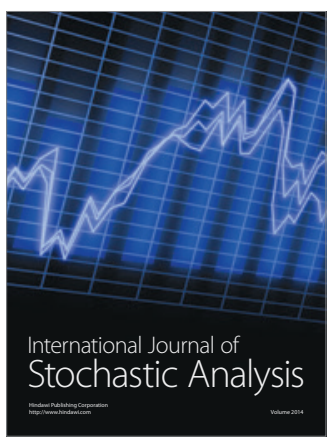

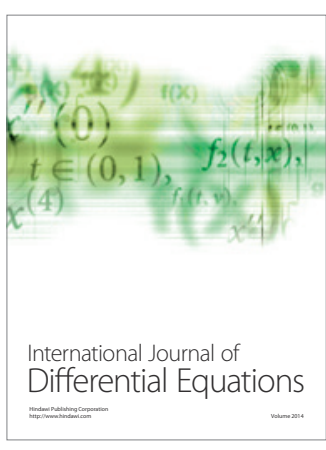
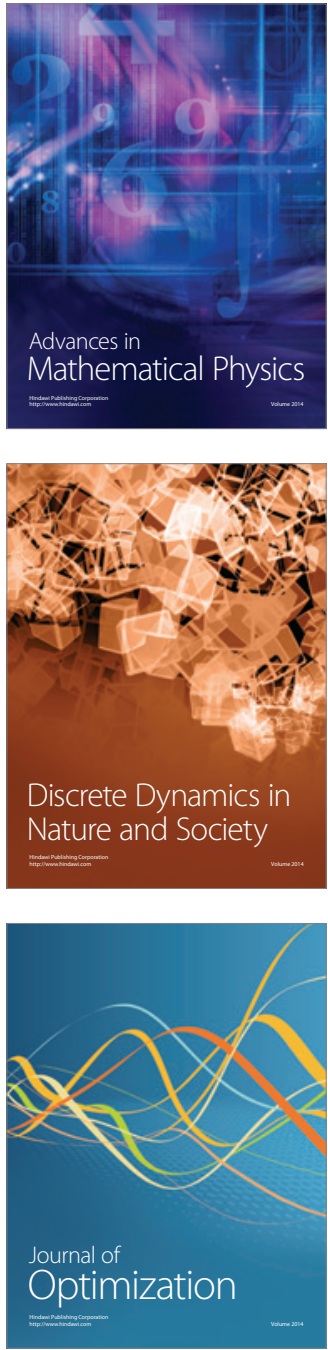